\title{
Highly sensitive detection of sentinel lymph node metastasis of breast cancer by digital PCR for RASSF1A methylation
}

\author{
MIZUHO ABE, NAOFUMI KAGARA, TOMOHIRO MIYAKE, TOMONORI TANEI, YASUTO NAOI, \\ MASAFUMI SHIMODA, KENZO SHIMAZU, SEUNG JIN KIM and SHINZABURO NOGUCHI \\ Department of Breast and Endocrine Surgery, Osaka University Graduate School of Medicine, \\ Suita, Osaka 565-0871, Japan
}

Received June 4, 2019; Accepted September 19, 2019

DOI: $10.3892 /$ or.2019.7363

\begin{abstract}
One-step nucleic acid amplification (OSNA) targeting cytokeratin 19 (CK19) mRNA expression and pathological examination are widely used for the intraoperative diagnosis of sentinel node (SN) metastasis. The aim of the present study was to develop a novel assay for detecting SN metastasis by targeting Ras association domain-containing protein 1 (RASSF1A) methylation in tumor cells, and to compare its performance with OSNA. Using digital PCR with methylation-specific restriction enzymes (RE-dMSP), our assay was able to detect $\geq 3$ copies of methylated DNA per well, and was $\geq 10$ times more sensitive than real-time PCR with bisulfite modification. OSNA lysates were examined using RE-dMSP and digital PCR for PIK3CA mutation, in the event that primary tumors were PIK3CA mutation-positive. RE-dMSP revealed a high concordance of $95.0 \%(153 / 161)$ with OSNA, and 100\% (59/59) with PIK3CA mutation for detecting SN metastasis. In 11 breast cancer cell lines, the variation in methylated RASSF1A copy number was significantly lower than that of CK19 mRNA (2.8 vs. 10.5-fold; $\mathrm{P}<0.01)$. RE-dMSP has the potential to more accurately detect $\mathrm{SN}$ metastasis, and to more precisely estimate total tumor loads in $\mathrm{SN}$, compared with OSNA.
\end{abstract}

\section{Introduction}

Sentinel lymph node (SN) biopsy is widely used to determine axillary lymph node (LN) status in clinically node-negative breast cancer patients $(1,2)$. In practice, $\mathrm{SN}$ metastasis is detected by intraoperative histopathological examination of frozen section(s) or cytological observation of touch imprints,

Correspondence to: Dr Naofumi Kagara, Department of Breast and Endocrine Surgery, Osaka University Graduate School of Medicine, 2-2-E10 Yamadaoka, Suita, Osaka 565-0871, Japan

E-mail:kagaran@onsurg.med.osaka-u.ac.jp

Key words: sentinel lymph node, molecular diagnosis, RASSF1A promoter methylation, one-step nucleic acid amplification lysate, breast cancer, digital PCR and is confirmed by postoperative pathological examination of permanent sections $(3,4)$. One-step nucleic acid amplification (OSNA) can be used to detect SN metastasis through the amplification of cytokeratin 19 (CK19) mRNA (which is expressed in tumor cells, but not normal cells of LNs) with the same accuracy as routine pathological examination (5). OSNA is also used to determine total tumor load (TTL) in SNs as the sum of CK19 mRNA copies, which is reportedly useful for predicting non-SN metastatic status $(6,7)$, as well as patient prognosis (8).

However, TTL determination by OSNA does not always accurately reflect the total number of tumor cells in the $\mathrm{SN}$, since the copy number of CK19 mRNAs per tumor cell varies considerably. In fact, it is reported that OSNA predicts a 30-fold difference in CK19 mRNA copies among tumors of the same size (9). By contrast, the amount of DNA per tumor cell is thought to be less variable, thus the detection of SN tumor cells from tumor-derived DNA is considered to more accurately determine TTL.

Ras association domain-containing protein 1 (RASSF1A) promoter methylation is one of the most frequently observed epigenomic changes in breast cancer $(10,11)$. Methylation-specific PCR (MSP) following bisulfite treatment is widely used to quantify methylated DNA. However, bisulfite treatment often results in considerable DNA loss $(12,13)$, and requires specialized optimization for digital PCR (dPCR) (14). A novel dPCR technique for the detection of methylated DNA was recently reported, using a methylation-specific restriction enzyme without bisulfite treatment (15-17). The aim of the present study was to develop a highly sensitive dPCR assay to detect RASSF1A methylation following restriction enzyme digestion (RE-dMSP), for the detection of tumor-derived methylated RASSF1A in SN lysates.

\section{Materials and methods}

Patients and samples. A total of 87 patients with breast cancer who underwent surgery with sentinel lymph node biopsy (SNB), and whose SNs were examined by OSNA at Osaka University between November 2015 and April 2017, were retrospectively included in this study (Fig. 1). The study was approved by the Ethical Review Board of Osaka University Hospital (approval date/number: 14 Aug 2014/\#14111), and informed consent 
was obtained from each patient. Of the 87 patients, 10 were excluded due to a lack of OSNA lysates, and six were excluded due to the lack of RASSF1A methylation in their primary tumors. Ultimately, $161 \mathrm{LNs}$ from 71 patients were included, and 166 lysates were analyzed (the LN was separated into two lysates in three SNs, and three lysates in one SN, due to its large size). SNB was performed with a combination of dye (patent blue and/or indocyanine green) and radiocolloid (technetium-99m tin colloid) or dye alone. A 1-mm-thick slice was cut from the center of each $\mathrm{SN}$ and intraoperatively subjected to frozen section analysis. The remaining LN tissue was used for OSNA, where the SN was homogenized in $4 \mathrm{ml}$ Lynorhag solution (Sysmex Corporation), of which $20 \mu$ lysate was used. The remaining lysate was stored at $-80^{\circ} \mathrm{C}$ until use. The CK19 copy number per assay was classified as follows: $>5,000,(++)$; $>250$ and $\leq 5,000,(+) ;>0$ and $\leq 250,(-)$; and 0 , (N.D.). OSNA (++) and (+) were considered to be positive, and isolated tumor cells (ITCs) were considered negative for $\mathrm{SN}$ metastasis.

Detection of RASSF1A methylation using RE-dMSP. DNA was extracted from $100 \mu \mathrm{l}$ SN lysate using the QIAamp Circulating Nucleic Acid Kit (Qiagen $\mathrm{GmbH}$ ) and eluted in $50 \mu \mathrm{l}$ desalted water. DNA solution $(6.6 \mu \mathrm{l})$ was incubated for $16 \mathrm{~h}$ at $37^{\circ} \mathrm{C}$ in a final volume of $20 \mu \mathrm{l}$, containing $1 \mathrm{X}$ ddPCR Supermix for probes (Bio-Rad Laboratories, Inc.), $900 \mathrm{nM}$ each primer, $250 \mathrm{nM}$ probe and $10 \mathrm{U}$ HhaI, HpaII (New England BioLabs, Inc.) and BstUI (Thermo Fisher Scientific, Inc.) each. These three methylation-sensitive restriction enzymes were selected since they can be used at the same incubation temperature $\left(37^{\circ} \mathrm{C}\right)$. The reaction time was set to $16 \mathrm{~h}(16,17)$ to allow for the complete digestion of unmethylated DNA. Methylation analysis was performed using three wells per assay. As a control to confirm the presence of DNA, the DNA solution $(2.0 \mu \mathrm{l})$ was also incubated without restriction enzymes. The primers (18) and probe (Universal ProbeLibrary \#19; cat. no. 04686926001; Roche Diagnostics $\mathrm{BmbH})$ are presented in Fig. 2 and Table SI. After incubation, droplet generation oil was added, and the mixture was loaded onto a QX100 droplet generator (Bio-Rad Laboratories, Inc.). Then, $40 \mu \mathrm{l}$ emulsified mixture was subjected to PCR using a T100 thermal cycler (Bio-Rad Laboratories, Inc.) under the following conditions: $95^{\circ} \mathrm{C}$ for $10 \mathrm{~min}$, followed by 40 cycles at $94^{\circ} \mathrm{C}$ for $30 \mathrm{sec}$ and $60^{\circ} \mathrm{C}$ for $1 \mathrm{~min}$, and $98^{\circ} \mathrm{C}$ for $10 \mathrm{~min}$. The data were analyzed using the QX100 droplet reader and QuantaSoft software version 1.7.4 (both Bio-Rad Laboratories, Inc.). The presence of $\geq 2$ dots/well was regarded as a positive result, and the copy numbers of three positive wells were totaled. The results for each $\mathrm{SN}$ divided into multiple lysates were summed.

For methylation analysis of primary breast tumors, DNA was extracted from three $10-\mu \mathrm{m}$ formalin-fixed paraffin-embedded (FFPE) tumor sections using the QIAamp DNA FFPE kit (Qiagen $\mathrm{GmbH}$ ), and RE-dMSP was performed. For sensitivity analysis of RE-dMSP, 0, 1, 3, 10, 30 and 100 copies of methylated DNA template (EpiScope ${ }^{\circledR}$ Methylated HeLa gDNA; Takara Bio, Inc.) spiked in 10,000 copies of unmethylated DNA from the peripheral blood leukocytes of a healthy individual were subjected to RE-dMSP with or without restriction enzymes. Conventional MSP with real-time PCR after bisulfite modification (qMSP) was performed as previously reported (19). The initial amount of DNA before bisulfite treatment was adjusted so that the input DNA copy number per well was the same as that of RE-dMSP. The sensitivity and positive detection rates were compared between the RE-dMSP and qMSP assays over eight wells.

Mutational analysis of PIK3CA in SNs and primary tumors. For the mutational analysis of primary breast tumors, DNA extracted from the FFPE tissue sections was subjected to real-time PCR analysis to detect the PIK3CA-H1047R mutation, as previously reported (20). For PIK3CA-mutation detection in SNs, DNA was extracted from $100 \mu 1$ SN lysate and eluted in $50 \mu \mathrm{l}$ desalted water. Then, $9 \mu \mathrm{l}$ DNA solution was subjected to QuantStudio ${ }^{\mathrm{TM}}$ 3D dPCR (Thermo Fisher Scientific, Inc.) (20). The sequences of the primers and probes are displayed in Table SI.

Estimation of DNA fragment size of methylated RASSF1A in SN tissues. DNA was extracted from $100 \mu \mathrm{l} \mathrm{SN}$ lysate and eluted with $50 \mu \mathrm{l}$ desalted water. To estimate the fragment size of the methylated DNA, $14 \mu 1$ DNA from each SN lysate was electrophoresed on a $2 \%$ agarose gel, and subsequently separated into short $(<500 \mathrm{bp})$ and long $(>500 \mathrm{bp})$ fragments. The DNA was extracted from each fraction using the QIAquick Gel Extraction Kit (Qiagen $\mathrm{GmbH}$ ) and RE-dMSP was performed.

Immunohistochemistry analysis of CK19. The expression of CK19 protein in primary breast tumors was assessed using immunohistochemistry with $4-\mu \mathrm{m}$ FFPE tissue sections. Immunohistochemical staining of each section was performed as previously described (21) with mouse monoclonal anti-CK19 primary antibody (clone, RCK 108; 1:50; Dako; Agilent Technologies, Inc.) and a peroxidase-conjugated secondary antibody [cat. no. 414131F; Histofine Simple Stain MAX PO (M); Nichirei Biosciences, Inc.]. Finally, the sections were visualized with 3,3-diaminobenzidine tetrahydrochloride (Wako Pure Chemical Industries, Ltd.) and counterstained with hematoxylin.

Quantification of methylated RASSF1A and CK19 mRNA in breast cancer cell lines. A total of six breast cancer cell lines (MCF7, MDA-MB-361, BT474, MDA-MB-453, MDA-MB-231 and BT20) were cultured in DMEM (Sigma-Aldrich; Merck KGaA), and five (ZR75-1, T47D, ZR75-30, SKBR3 and AU565) were cultured in RPMI-1640 (Sigma-Aldrich; Merck KGaA) at $37^{\circ} \mathrm{C}\left(5 \% \mathrm{CO}_{2}\right)$ in a humidified atmosphere. DNA and mRNA were extracted from $10 \mathrm{E}+6$ cells from each cell line using the DNeasy Blood \& Tissue Kit and the RNeasy Mini Kit (both Qiagen $\mathrm{GmbH}$ ), respectively. The DNA was subjected to RE-dMSP, and the copy number of methylated RASSF1A per cell was obtained. Briefly, $1 \mu \mathrm{g}$ total RNA was reverse-transcribed into cDNA using the ReverTra Ace ${ }^{\circledR}$ qPCR RT kit (Toyobo Life Science), and CK19 mRNA expression was assessed using the Light Cycler 480 Real-time PCR System (Roche Applied Science) with the following conditions: $95^{\circ} \mathrm{C}$ for $10 \mathrm{~min}$, followed by 50 cycles at $95^{\circ} \mathrm{C}$ for $15 \mathrm{sec}$ and $60^{\circ} \mathrm{C}$ for $60 \mathrm{sec}$, with a final cycle at $50^{\circ} \mathrm{C}$ for $10 \mathrm{sec}$. KRT19 (CK19) TaqMan ${ }^{\circledR}$ Gene Expression Assays (Hs01051611_gH; Applied Biosystems; Thermo Fisher Scientific, Inc.) were used to 


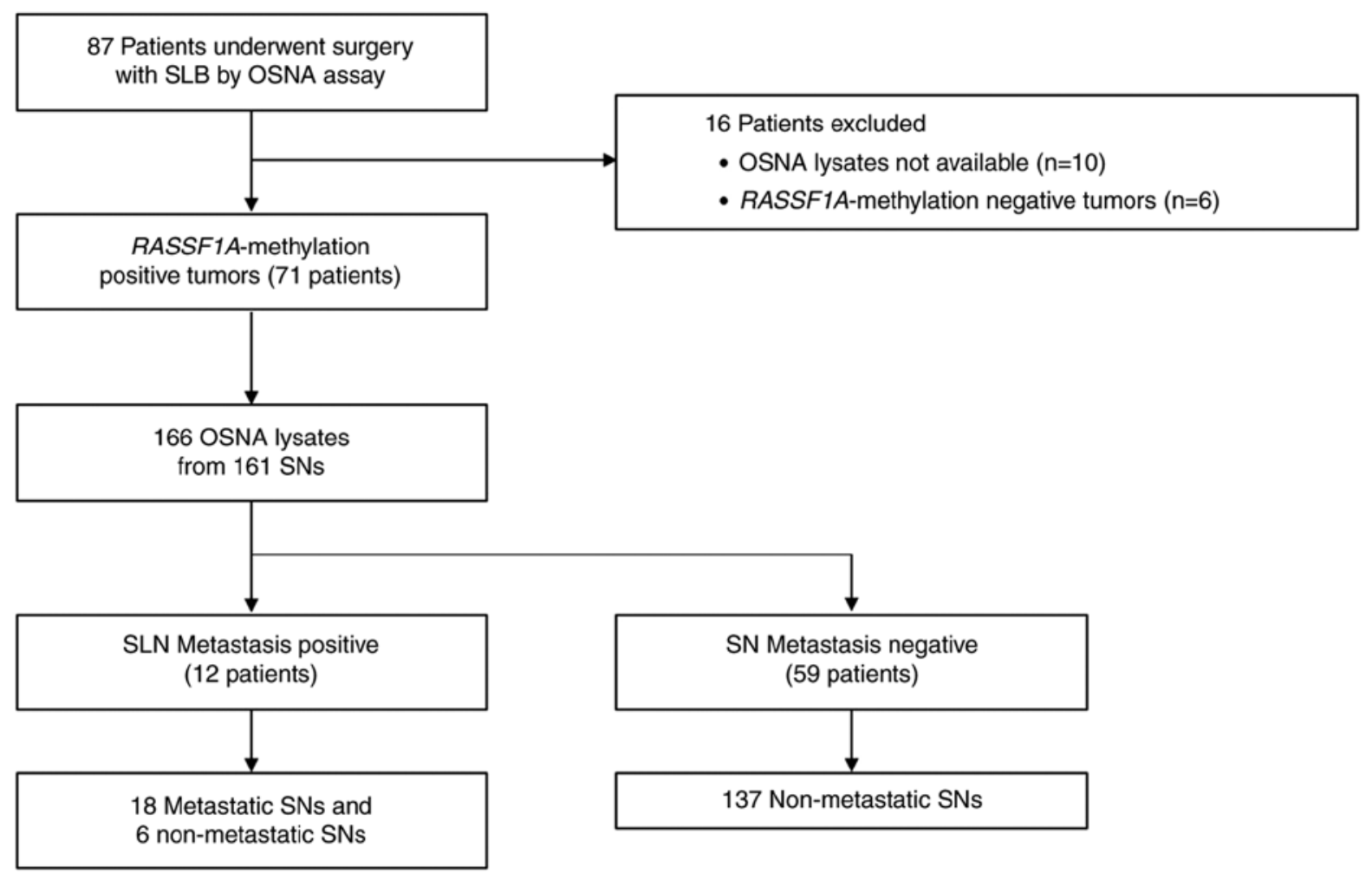

Figure 1. Flowchart of the patient selection process for detecting methylated RASSF1A in SNs. RASSF1A, Ras association domain-containing protein 1; SN, sentinel node.

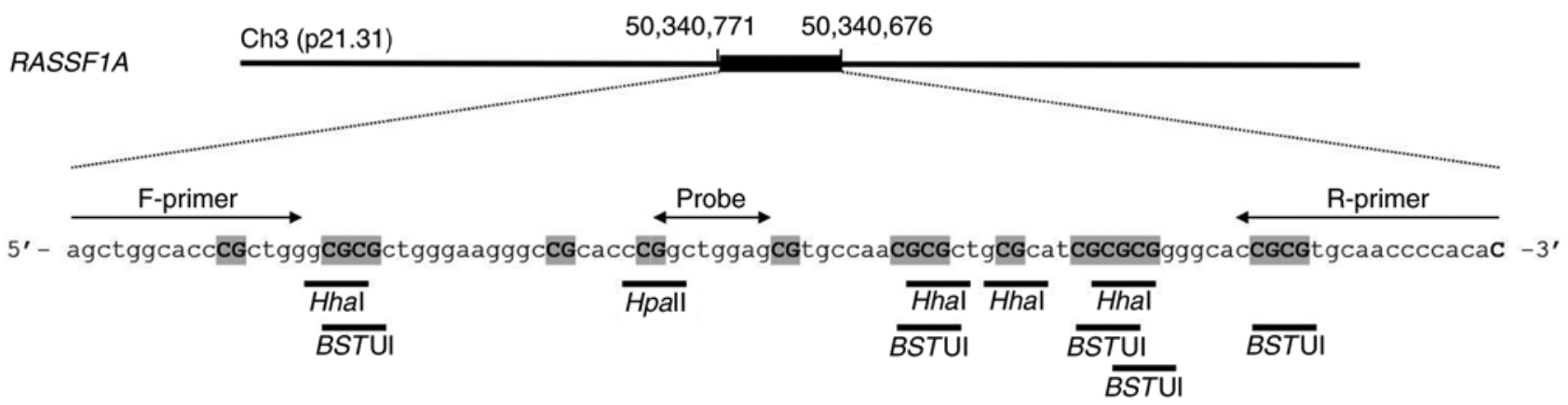

Figure 2. Schematic of the primers and probe used for RE-dMSP of the RASSF1A promoter. The location of the primers and probe, and the recognition sites of three methylation-sensitive restriction enzymes ( $\mathrm{HpaII}$, HhaI and Bst $\mathrm{UI}$ ) are presented. RE-dMSP, PCR with methylation-specific restriction enzymes; RASSF1A, Ras association domain-containing protein 1.

conduct real-time PCR. The relative CK19 mRNA expression level per cell was obtained, and a PCR product was used as the standard.

Statistical analysis. Statistical analyses were performed using JMP Pro 11.2.0 (SAS Institute, Inc.) or GraphPad Prism 6 software (GraphPad Software, Inc.). The association between clinicopathological parameters and the copy number of methylated DNA or CK19 mRNA in SNs was evaluated using Fisher's exact test. Associations between the copy number of methylated DNA and CK19 mRNA in lysates were evaluated using the Wilcoxon signed-rank sum test. Differences in the copy number ranges of methylated DNA and CK19 mRNA among breast cancer cell lines were evaluated using the F-test.

\section{Results}

Sensitivity of RE-dMSP. The sensitivity of RE-dMSP was evaluated for the detection of methylated RASSF1A, using $0,1,3,10,30$ and 100 copies of methylated DNA spiked in 10,000 copies of unmethylated DNA per well. A linear correlation was observed across all concentrations of methylated RASSF1A between the input copy number and the RE-dMSP results (Fig. 3A). No copies of methylated RASSF1A were detected in the $100 \%$ unmethylated DNA samples (data not shown), indicating that methylated RASSF1A was completely removed by restriction enzyme digestion. The assay results without restriction enzymes accurately represented the total amount of input DNA. The detection sensitivity of RE-dMSP in eight wells was $37.5,62.5$ and $100 \%$ for 1,3 and $\geq 10$ copies 
A

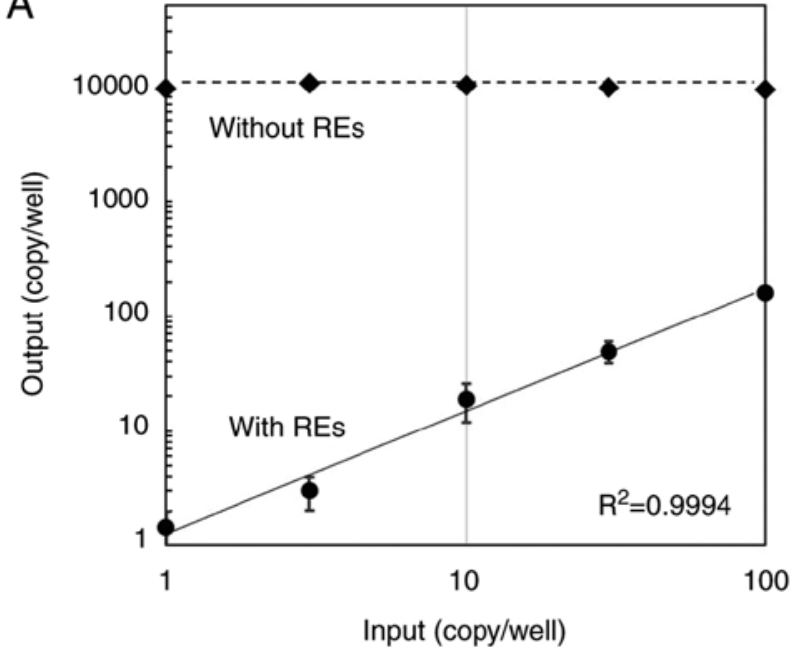

B

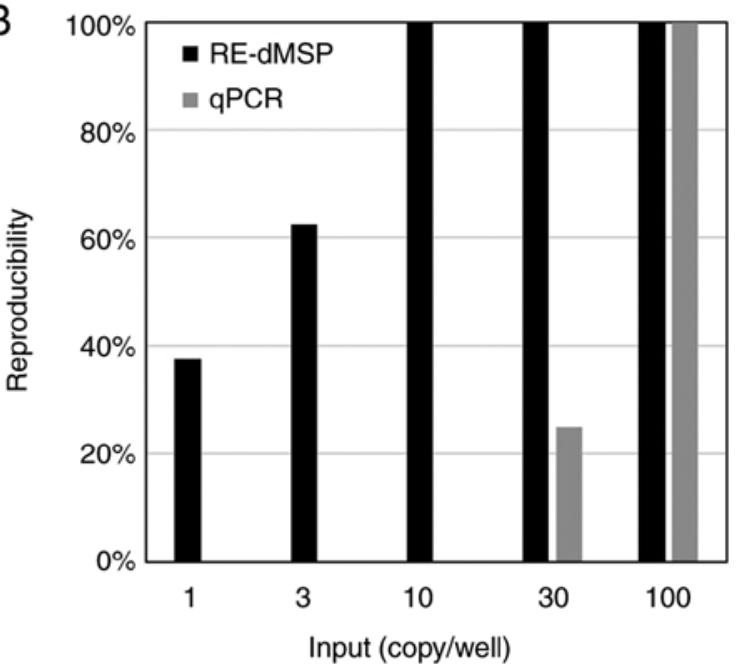

Figure 3. Sensitivity of RE-dMSP for the detection of methylated RASSF1A. (A) Detection sensitivity of RE-dMSP was assessed using $0,1,3,10,30$ and 100 copies of methylated genomic DNA, spiked in 10,000 copies of unmethylated genomic DNA extracted from the peripheral blood leukocytes of a healthy individual. Methylated RASSF1A was quantified by RE-dMSP with restriction enzymes (solid line, with REs), and the total inputs of methylated and unmethylated DNA were measured without restriction enzymes (dotted line, without REs). Error bars indicate the standard deviation of eight experiments. (B) Positive detection rate in eight experiments for each sample, compared between RE-dMSP and qPCR with bisulfite modification. RE-dMSP, dPCR with methylation-specific restriction enzymes; RASSF1A, Ras association domain-containing protein 1; RE, restriction enzyme; qPCR, quantitative PCR.

of methylated DNA, respectively, while that of qMSP was 0,25 and $100 \%$ for $\leq 10,30$ and 100 copies of methylated DNA, respectively. This indicated that RE-dMSP was $\geq 10$ times more sensitive than conventional qMSP following bisulfite modification (Fig. 3B). Considering the probability distribution of methylated DNA in the templates (according to the binominal model), the sensitivity of RE-dMSP was estimated to be between two and three copies per well.

$R E$-dMSP using SN lysates for OSNA. Using the primary tumor samples of 77 breast cancer patients who underwent SNB and OSNA, RASSF1A methylation was screened by RE-dMSP; $71(92.2 \%)$ of the samples were revealed to exhibit RASSF1A methylation (Fig. 1). The patient clinicopathological characteristics are presented in Table I. Of these 71 patients, 12 (16.9\%) possessed SN metastases. In total, $161 \mathrm{SNs}$ from these 71 patients were analyzed using OSNA, including 18 positive and 143 negative SNs. Among the 161 SNs, RASSF1A-methylation analysis was performed by RE-dMSP, and methylation was detected in 22 SNs from 14 patients. The amount of total DNA in the SN lysates ranged from 1,600 to 1,593,000 copies per $100 \mu \mathrm{l}$, confirming successful DNA extraction from all samples. Methylated RASSF1A was observed significantly more frequently in patients with large tumors that exhibited positive lymphovascular invasion (Table I). The expression levels of CK19 mRNA also exhibited a similar trend, although the difference was not significant. The relationship between the amounts of methylated RASSF1A and CK19 mRNA in SNs is presented in Fig. 4A. Methylated RASSF1A was detected in all of the SNs in which CK19 mRNA was highly expressed [OSNA (++)] (range, 9.8-95,000 copies/assay; $n=13$ ), and methylated RASSF1A was not present in SNs in which CK19 mRNA was not detected [OSNA (N.D.); n=91] (Fig. 4B). Methylated RASSF1A was detected in three of the five OSNA (+) SNs $(60 \%$; range, 7.4-12,800 copies/assay), and in six of the 52 OSNA (-) SNs (11.5\%; range, 19.8-348 copies/assay). The concordance rate between the methylated RASSF1A status and the OSNA results was $95.0 \%$ (153/161). In the six RASSF1A-methylation (+) and OSNA (-) SNs from five patients (one patient possessed two SNs), immunohistochemistry was used to assess CK19 protein expression in the primary tumors; the results revealed strong-positive staining in all five patient samples (Fig. S1).

DNA fragment size of methylated RASSF1 in SNs. It has previously been reported that circulating tumor DNA (ctDNA) generated in primary tumors can be detected in SNs (22). ctDNA is segmented into $<180 \mathrm{bp}$ fragments by apoptosis, and can therefore be detected by the short amplicon $\mathrm{dPCR}$ product (96 bp) of RE-dMSP $(23,24)$. By contrast, metastatic tumor cells in SNs can produce long DNA fragments of methylated RASSF1A. In the present study, DNA fragment size was assessed to determine whether the methylated RASSF1A detected in SNs was derived from tumor cells, or from methylated RASSF1A fragments from the primary tumor migrating through the lymphatic vessels. A total of six methylated RASSF1A-positive, CK19 mRNA-negative SNs were selected. The SN lysates were available from three of these SNs and subjected to the following experimental procedures: Total DNA extracted from the lysates was separated into short ( $<500 \mathrm{bp}$ ) and long ( $>500 \mathrm{bp}$ ) DNA fractions by agarose gel electrophoresis, and evaluated by RE-dMSP (Fig. S2A). Methylated RASSF1A was detected in the long DNA fractions of all three SNs, and in the short fractions of two SNs (Fig. S2B). Given that all methylated RASSF1A-positive SNs contained long DNA fragments, SN-associated methylated RASSF1A was considered to originate from tumor cells in the $\mathrm{SN}$, and not from the primary tumor.

Detection of SN metastasis by dPCR for the PIK3CA mutation. To further investigate whether the methylated RASSF1A was derived from unexpected methylation in non-tumor cells of the SN (including lymphocytes), mutational analysis of 
Table I. Association between patient clinicopathological parameters and RASSF1A methylation or CK19 mRNA expression in SNs.

\begin{tabular}{|c|c|c|c|c|c|c|c|}
\hline \multirow[b]{2}{*}{ Variables } & \multirow[b]{2}{*}{$\mathrm{n}$} & \multicolumn{2}{|c|}{$\begin{array}{c}\text { RASSF1A } \\
\text { methylation in SN }\end{array}$} & \multirow[b]{2}{*}{ P-value } & \multicolumn{2}{|c|}{$\begin{array}{c}\text { CK19 mRNA } \\
\text { in SN }\end{array}$} & \multirow[b]{2}{*}{ P-value } \\
\hline & & Positive & Negative & & Positive & Negative & \\
\hline Total patients & & 71 & 14 & 57 & & 12 & 59 \\
\hline \multicolumn{8}{|l|}{ Age } \\
\hline$<50$ & 23 & 7 & 16 & 0.107 & 5 & 18 & 0.332 \\
\hline$\geq 50$ & 48 & 7 & 41 & & 7 & 41 & \\
\hline \multicolumn{8}{|c|}{ Tumor size (mm) } \\
\hline$<20$ & 40 & 4 & 36 & 0.033 & 3 & 37 & 0.025 \\
\hline$\geq 20$ & 31 & 10 & 21 & & 9 & 22 & \\
\hline \multicolumn{8}{|c|}{ Histological grade } \\
\hline 1,2 & 53 & 11 & 42 & 0.332 & 11 & 42 & 0.128 \\
\hline 3 & 18 & 3 & 15 & & 1 & 17 & \\
\hline \multicolumn{8}{|l|}{ LVI } \\
\hline Positive & 7 & 5 & 2 & 0.003 & 3 & 4 & 0.089 \\
\hline Negative & 64 & 9 & 55 & & 9 & 55 & \\
\hline \multicolumn{8}{|l|}{ ER/PgR } \\
\hline Positive & 65 & 13 & 52 & 0.663 & 11 & 54 & 0.685 \\
\hline Negative & 6 & 1 & 5 & & 1 & 5 & \\
\hline \multicolumn{8}{|l|}{ HER 2} \\
\hline Positive & 5 & 0 & 5 & 0.322 & 0 & 5 & 0.385 \\
\hline Negative & 66 & 14 & 52 & & 12 & 54 & \\
\hline \multicolumn{8}{|l|}{ Recurrence } \\
\hline Positive & 0 & 0 & 0 & - & 0 & 0 & - \\
\hline Negative & 71 & 14 & 57 & & 12 & 59 & \\
\hline
\end{tabular}

RASSF1A, Ras association domain-containing protein 1; CK19, cytokeratin 19; LVI, lymphovascular invasion; ER, estrogen receptor; PgR, progesterone receptor; HER2, human epidermal receptor 2.

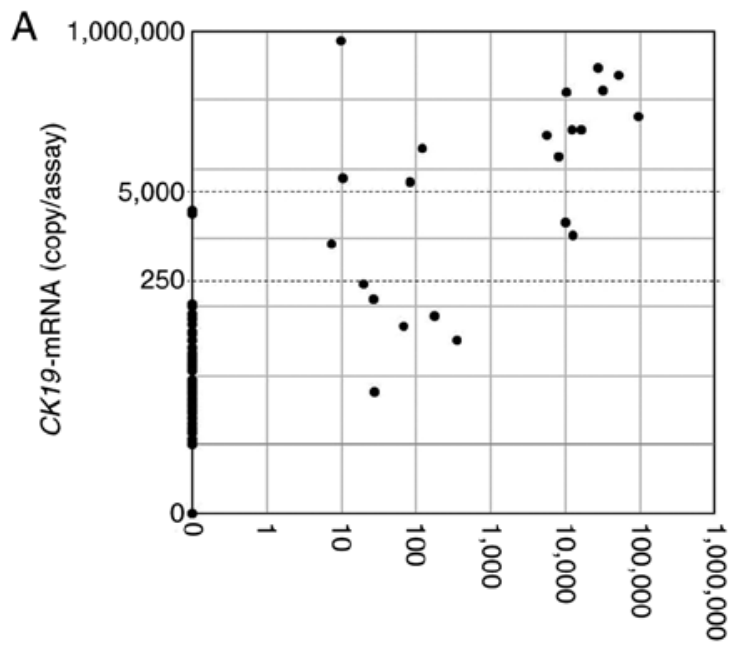

Methylated RASSF1A (copy/assay)

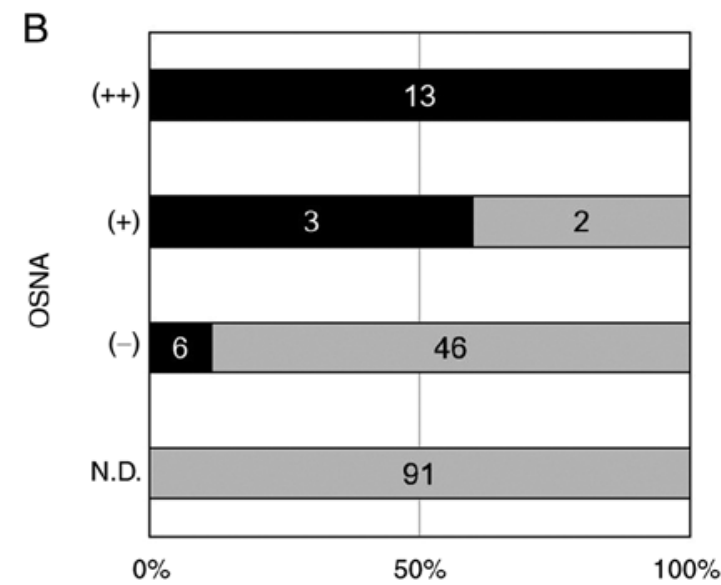

Methylated RASSF1A (+)

Methylated RASSF1A (-)

Figure 4. Association between the methylated RASSF1A copy number and CK19 mRNA expression in SN lysates of OSNA. (A) Correlation between the copy number (copies/assay) of methylated RASSF1A as determined by RE-dMSP, and CK19 mRNA expression as determined by OSNA. (B) The number of methylated RASSF1A-positive and -negative SNs is presented according to OSNA diagnoses; CK19 mRNA >5,000, (++); >250 and $\leq 5,000,(+) ;>0$ and $\leq 250$, (-); 0, (N.D.). RASSF1A, Ras association domain-containing protein 1; SN, sentinel node; CK19, cytokeratin 19; OSNA, one-step nucleic acid amplification; RE-dMSP, dPCR with methylation-specific restriction enzymes. 


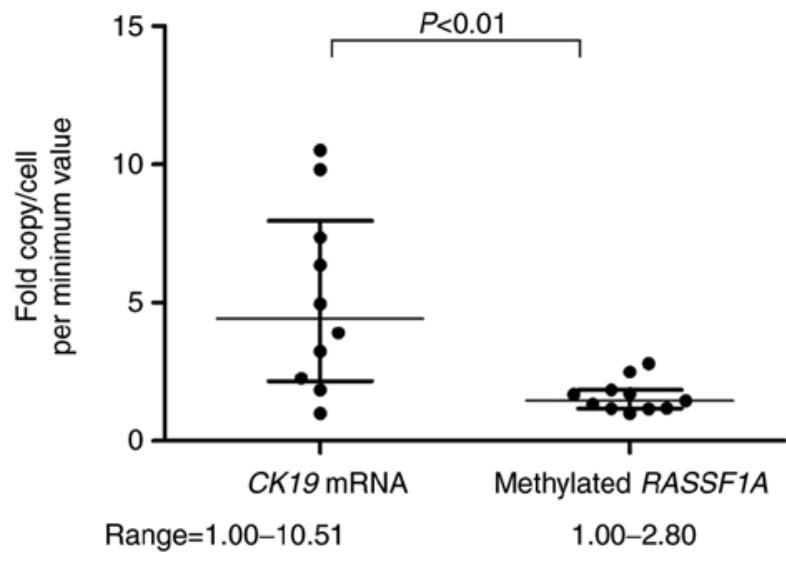

Figure 5. Variation in the copy number of methylated RASSF1A and CK19 mRNA expression in 11 breast cancer cell lines. Fold difference in the copy number of methylated RASSF1A and CK19 mRNAs per cell according to the minimum value among the cell lines; the variation in fold copy number was compared between CK19 mRNA and methylated RASSF1A (F-test). Bars indicate the median and interquartile values. The MBA-MB-231 cell line was excluded from CK19 mRNA analysis due to a lack of CK19 expression. RASSF1A, Ras association domain-containing protein 1; CK19, cytokeratin 19.

the SN lysates was performed, targeting a mutation specific to primary tumors (which does not occur in non-cancerous cells) (25). The PIK3CA H1047R mutation was used in this study, since it is one of the most frequently observed mutations in breast cancer $(26,27)$. A total of 71 tumors were screened using real-time PCR, and 22 were revealed to possess the mutation. A total of $59 \mathrm{SN}$ lysates from these 22 patients were subjected to dPCR analysis for the PIK3CA mutation, which was detected in 11 SNs (18.6\%; range, 6.5-6,106.9 copies/assay; Fig. S3A). Methylated RASSF1A was detected in all of the 11 PIK3CA mutation-positive SNs, but not in the remaining 48 mutation-negative SNs, indicating complete agreement between mutation and methylation status (Fig. S3B).

Association between methylated RASSF1A and CK19 mRNA expression in breast cancer cells. CK19 mRNA expression and the presence of methylated RASSF1A alleles were analyzed in 11 breast cancer cell lines. An extremely low level of CK19 mRNA expression was detected in MDA-MB-231 cells (20 copies/cell), which were considered to be CK19 negative. In the other 10 cell lines, the expression level ranged from 3,224 to 33,877 copies/cell, which equated to a 10.5-fold difference (Fig. 5). By contrast, the copy numbers of methylated RASSF1A alleles were in the range of 0.52-1.44 copies/cell, exhibiting a 2.80-fold difference. The fold difference in copy number per cell was significantly lower for RASSF1A methylation than for CK19 mRNA, indicating that methylated DNA alleles more precisely reflect the number of tumor cells.

\section{Discussion}

In order to detect SN tumor-derived DNA, an RE-dMSP assay was developed to accurately measure RASSF1A methylation using dPCR following restriction enzyme digestion. RE-dMSP was able to detect as few as three copies of methylated RASSF1A by complete digestion of unmethylated DNA, that corresponds to 150 tumor cells per node, showing a sensitivity $>10$ times greater than that of the bisulfite method. A highly linear correlation between the RE-dMSP results and the amount of input DNA also ensured accurate and quantitative measurement of SN tumor cells.

The RE-dMSP assay, which was conducted with $161 \mathrm{SN}$ lysates, demonstrated a high concordance of $95 \%$ (153/161 SNs) with OSNA; eight discordant cases were found, including six OSNA (-)/methylation (+) and two OSNA (+)/methylation (-) SNs. The fact that the PIK3CA mutation status in the SN lysates revealed complete agreement with the RASSF1A methylation status indicates that non-tumorous cells in the lymph nodes do not exhibit RASSF1A methylation, because, if they did, a considerable amount of RASSF1A methylation would have been detected in PIK3CA mutation-negative SNs. Therefore, it is surmised that the six OSNA (-)/methylation (+) SNs are unlikely to have been RE-dPCR false-positives, and were more likely to be OSNA false-negatives. Since CK19 protein expression was confirmed in the primary tumors of all patients, most of these false-negatives are unlikely to be attributable to low CK19 mRNA expression within the tumor cells, although the possibility of low CK19 mRNA expression in CK19 protein-positive tumors still remains (9).

In addition, the DNA fragment size of methylated RASSF1A in the OSNA (-)/methylation (+) SN group was analyzed, in order to determine whether methylated RASSF1A originated from metastatic tumor cells in the SNs, or from primary tumors via the lymphatic vessels. Taking advantage of the fact that methylated RASSF1A from primary tumors has a short DNA fragment size $(<500 \mathrm{bp}$; as it is generated from apoptosis), while that from metastatic tumor cells may be either short or long (>500 bp), the origin of the methylated RASSF1A was distinguished by analyzing DNA fragment size. In the present study, the presence of long DNA fragments was indicated in all three of the analyzed SNs, confirming the presence of tumor cells in SNs. Thus, it is highly likely that OSNA (-)/methylation (+) SNs reflect tumor metastases, and thus represent false-negatives from OSNA.

Only two of the SNs were OSNA (+)/methylation (-), suggesting the possibility of false-negatives from RE-dMSP. However, in addition to being negative for RASSF1A methylation, these two SNs were also PIK3CA mutation-negative, although the corresponding primary tumors were positive for the PIK3CA mutation. Since RE-dMSP is sensitive enough to detect only a few copies per assay, it is unlikely that tumor cells in the SNs were missed by RE-dMSP; it is more likely that OSNA resulted in false-positives.

The TTL in SNs has been reported to correlate with the extent of non-SN metastases (28), and CK19 mRNA copies measured by OSNA have been used to estimate TTL in several predictive models $(6,7)$. However, there is an $\sim 30$-fold difference in CK19 mRNA expression among breast tumors (9). In line with this, the present study also demonstrated a 10.8-fold difference in CK19 mRNA expression per cell in 10 breast cancer cell lines (plus one cell line that was CK19-negative). By contrast, the fold difference in the methylated RASSF1A copy number was as low as 2.8 among these 11 cell lines. This difference was assumed to result from a loss of heterozygosity (29) or aneuploidy (30-32). These results indicate that methylated RASSF1A can predict TTL more accurately than CK19 mRNA expression levels. At present, SN micrometastases 
(equivalent to ITCs) are considered to have little significance in prognosis (33). However, in these previous studies, SN metastases were usually evaluated by histological examination of a few representative sections of each $\mathrm{SN}$, rather than a series of sections from each SN. On the other hand, RE-dMSP can detect metastases in each entire SN, thus quantification of TTL in each $\mathrm{SN}$ is considered to be more accurate than histological examination. Thus, it is possible that future RE-dMSP studies may disclose a new prognostic value for small SN metastases which are not detectable by histological examination.

A possible limitation of the present study is that RASSF1A methylation is not observed in all breast cancers, and its expression in the primary tumor is a prerequisite for RE-dMSP. In the present study, as many as $92.2 \%$ of breast tumors were RASSF1A methylation-positive; this was consistent with previous studies reporting frequencies of $90.4-97.8 \%(10,19)$, suggesting that RE-dMSP is applicable for use in $>90 \%$ of breast tumors. Moreover, we had already reported that $\geq 1$ of the three RASSF1A, GSTP1 and RARB2 genes is methylated in $98 \%$ of breast tumors, indicating that for RE-dMSP, the addition of GSTP1 and RARB2 to RASSF1A would enhance its applicability to nearly all breast tumors $(10,34)$. OSNA has been repeatedly revealed to be as accurate as routine histological examination for the detection of SN metastases in unselected breast tumors (5), and is used in clinical practice in numerous countries. A lack of CK19 mRNA expression (a target of OSNA) has been reported in 1.6-3.0\% of breast tumors $(35,36)$. These results suggest that RE-dMSP targeting methylated GSTP1, RARB2 and RASSF1A genes may also be applicable to unselected breast tumors, much like OSNA. Further studies to pursue this possibility would be worthwhile.

The second limitation is that RE-dMSP cannot be used for the intraoperative diagnosis of SN metastasis, since it requires an overnight assay procedure. We believe that OSNA and RE-dMSP are complementary to each other for the detection of SN metastasis: OSNA is quicker than RE-dMSP and thus suitable for intraoperative analysis, while RE-dMSP provides a more accurate assessment of TTL, and is thus more suitable for postoperative analyses. The association between OSNA and RE-dMSP is analogous to that between intraoperative frozen and postoperative FFPE section analyses. Therefore, if the clinical significance of TTL determined by RE-dMSP is confirmed in the future, RE-dMSP may potentially be used alongside OSNA in daily practice, replacing the need for histological analysis. The third limitation was our limited sample size, which included only $161 \mathrm{LNs}$ from 71 patients. We are currently working with other institutions to increase the sample population and hope to corroborate the findings in the future.

In conclusion, the present study demonstrated the development of an RE-dMSP assay to precisely detect RASSF1A methylation by methylation-specific restriction enzyme digestion, followed by dPCR. RE-dMSP was indicated to detect SN metastasis more accurately, and to estimate TTL more precisely than OSNA. However, the clinical utility of RE-dMSP requires further validation, including future studies with a greater number of patients.

\section{Acknowledgements}

Not applicable.

\section{Funding}

No specific grants were received from funding agencies in the public, commercial or not-for-profit sectors. SN received honoraria and research funding from Sysmex for research not related to the present study, and holds joint patents with Sysmex on subjects not related to the present study. YN also received honoraria from Sysmex, and holds joint patents with Sysmex on subjects not related to the present study.

\section{Availability of data and materials}

The datasets used during the present study are available from the corresponding author upon reasonable request.

\section{Authors' contributions}

MA performed the experiments and analyzed the data. TM, TT, YN, MS, KS, and SJK collected the clinical samples. MA, NK and SN designed and drafted the manuscript. All authors read and approved the final manuscript and agree to be accountable for all aspects of the research in ensuring that the accuracy or integrity of any part of the work are appropriately investigated and resolved.

\section{Ethics approval and consent to participate}

The study was approved by the Ethical Review Board of Osaka University Hospital (approval date/number: 14 Aug 2014/\#14111), and informed consent was obtained from each patient.

\section{Patient consent for publication}

Not applicable.

\section{Competing interests}

This study was supported in part by the research funding from Novartis, Pfizer, and Sysmex. Shinzaburo Noguchi has received research grant for other study from AstraZeneca and honoraria from AsstraZeneca, Novartis, Pfizer, and Sysmex, and has been an advisor for AstraZeneca and Novartis. Naofumi Kagara received honoraria from AstraZeneca and Novartis. Yasuto Naoi received honoraria from AstraZeneca and Sysmex and research grant from AstraZeneca for other study. Masafumi Shimoda received honoraria from Novartis. Kenzo Shimazu received honoraria from AstraZeneca. Seung Jin Kim received honoraria from AstraZeneca, Novartis and Pfizer. The other authors declare that they do not have a financial relationship with the organizations that sponsored the research.

\section{References}

1. Lyman GH, Giuliano AE, Somerfield MR, Benson AB III, Bodurka DC, Burstein HJ, Cochran AJ, Cody HS III, Edge SB, Galper S, et al: American society of clinical oncology guideline recommendations for sentinel lymph node biopsy in early-stage breast cancer. J Clin Oncol 23: 7703-7720, 2005.

2. Lyman GH, Temin S,Edge SB, Newman LA, Turner RR, WeaverDL, Benson AB III, Bosserman LD, Burstein HJ, Cody H III, et al: Sentinel lymph node biopsy for patients with early-stage breast cancer: American Society of Clinical Oncology clinical practice guideline update. J Clin Oncol 32: 1365-1383, 2014. 
3. Veronesi U, Paganelli G, Viale G, Luini A, Zurrida S, Galimberti V, Intra M, Veronesi P, Maisonneuve P, Gatti G, et al: Sentinel-lymph-node biopsy as a staging procedure in breast cancer: Update of a randomised controlled study. Lancet Oncol 7: 983-990, 2006.

4. Krag DN, Anderson SJ, Julian TB, Brown AM, Harlow SP, Ashikaga T, Weaver DL, Miller BJ, Jalovec LM, Frazier TG, et al: Technical outcomes of sentinel-lymph-node resection and conventional axillary-lymph-node dissection in patients with clinically node-negative breast cancer: Results from the NSABP B-32 randomised phase III trial. Lancet Oncol 8: 881-888, 2007.

5. Tamaki Y: One-step nucleic acid amplification assay (OSNA) for sentinel lymph node biopsy. Breast Cancer 22: 230-234, 2015.

6. Shimazu K, Sato N, Ogiya A, Sota Y, Yotsumoto D, Ishikawa T, Nakamura S, Kinoshita T, Tsuda H, Ohi Y, et al: Intraoperative nomograms, based on one-step nucleic acid amplification, for prediction of non-sentinel node metastasis and four or more axillary node metastases in breast cancer patients with sentinel node metastasis. Ann Surg Oncol 25: 2603-2611, 2018.

7. Rubio IT, Espinosa-Bravo M, Rodrigo M, Amparo Viguri Diaz M, Hardisson D, Sagasta A, Dueñas B and Peg V: Nomogram including the total tumoral load in the sentinel nodes assessed by one-step nucleic acid amplification as a new factor for predicting nonsentinel lymph node metastasis in breast cancer patients. Breast Cancer Res Treat 147: 371-380, 2014.

8. Osako T, Iwase T, Ushijima M, Yonekura R, Ohno S and Akiyama F: A new molecular-based lymph node staging classification determines the prognosis of breast cancer patients Br J Cancer 117: 1470-1477, 2017.

9. Tsujimoto M, Nakabayashi K, Yoshidome K, Kaneko T, Iwase T, Akiyama F, Kato Y, Tsuda H, Ueda S, Sato K, et al: One-step nucleic acid amplification for intraoperative detection of lymph node metastasis in breast cancer patients. Clin Cancer Res 13: 4807-4816, 2007.

10. Yamamoto N, Nakayama T, Kajita M, Miyake T, Iwamoto T, Kim SJ, Sakai A, Ishihara H, Tamaki Y and Noguchi S: Detection of aberrant promoter methylation of GSTP1, RASSF1A and RAR $\beta 2$ in serum DNA of patients with breast cancer by a newly established one-step methylation-specific PCR assay. Breast Cancer Res Treat 132: 165-173, 2012.

11. Park SY, Kwon HJ, Lee HE, Ryu HS, Kim SW, Kim JH, Kim IA, Jung N, Cho NY and Kang GH: Promoter CpG island hypermethylation during breast cancer progression. Virchows Arch 458: 73-84, 2011.

12. Okamoto A: Chemical approach toward efficient DNA methylation analysis. Org Biomol Chem 7: 21-26, 2009.

13. Grunau C, Clark SJ and Rosenthal A: Bisulfite genomic sequencing: Systematic investigation of critical experimental parameters. Nucleic Acids Res 29: E65-E75, 2001

14. Li M, Chen WD, Papadopoulos N, Goodman SN, Bjerregaard NC, Laurberg S, Levin B, Juhl H, Arber N, Moinova H, et al: Sensitive digital quantification of DNA methylation in clinical samples. Nat Biotechnol 27: 858-863, 2009.

15. Wu Z, Bai Y, Cheng Z, Liu F, Wang P, Yang D, Li G, Jin Q, Mao H and Zhao J: Absolute quantification of DNA methylation using microfluidic chip-based digital PCR. Biosens Bioelectron 96: 339-344, 2017.

16. Suehiro Y, Zhang Y, Hashimoto S, Takami T, Higaki S, Shindo Y, Suzuki N, Hazama S, Oka M, Nagano H, et al: Highly sensitive faecal DNA testing of TWIST1 methylation in combination with faecal immunochemical test for haemoglobin is a promising marker for detection of colorectal neoplasia. Ann Clin Biochem 55: 59-68, 2018

17. Suehiro Y, Hashimoto S, Higaki S, Fujii I, Suzuki C, Hoshida T, Matsumoto T, Yamaoka Y, Takami T, Sakaida I and Yamasaki T: Blood free-circulating DNA testing by highly sensitive methylation assay to diagnose colorectal neoplasias. Oncotarget 9 : 16974-16987, 2018.

18. Kristiansen S, Nielsen D and Sölétormos G: Detection and monitoring of hypermethylated RASSF1A in serum from patients with metastatic breast cancer. Clin Epigenetics 8: 35, 2016.

19. Takahashi H, Kagara N, Tanei T, Naoi Y, Shimoda M, Shimomura A, Shimazu K, Kim SJ and Noguchi S: Correlation of methylated circulating tumor DNA with response to neoadjuvant chemotherapy in breast cancer patients. Clin Breast Cancer 17: 61-69.e3, 2017
20. Oshiro C, Kagara N, Naoi Y, Shimoda M, Shimomura A, Maruyama N, Shimazu K, Kim SJ and Noguchi S: PIK3CA mutations in serum DNA are predictive of recurrence in primary breast cancer patients. Breast Cancer Res Treat 150: 299-307, 2015.

21. Morimoto K, Kim SJ, Tanei T, Shimazu K, Tanji Y, Taguchi T, Tamaki Y, Terada N and Noguchi S: Stem cell marker aldehyde dehydrogenase 1-positive breast cancers are characterized by negative estrogen receptor, positive human epidermal growth factor receptor type 2 and high Ki67 expression. Cancer Sci 100: 1062-1068, 2009.

22. Miyamura Y, Kagara N, Miyake T, Tanei T, Naoi Y, Shimoda M, Shimazu K, Kim SJ and Noguchi S: Drainage of tumor-derived DNA into sentinel lymph nodes in breast cancer patients. Pathol Oncol Res. Feb 25, 2019 (Epub ahead of print).

23. Jahr S, Hentze H, Englisch S, Hardt D, Fackelmayer FO, Hesch RD and Knippers R: DNA fragments in the blood plasma of cancer patients: Quantitations and evidence for their origin from apoptotic and necrotic cells. Cancer Res 61: 1659-1665, 2001.

24. Heitzer E, Ulz P and Geigl JB: Circulating tumor DNA as a liquid biopsy for cancer. Clin Chem 61: 112-123, 2015.

25. Broderick DK, Di C, Parrett TJ, Samuels YR, Cummins JM, McLendon RE, Fults DW, Velculescu VE, Bigner DD and Yan H: Mutations of PIK3CA in anaplastic oligodendrogliomas, high-grade astrocytomas and medulloblastomas. Cancer Res 64: 5048-5050, 2004.

26. Cancer Genome Atlas Network: Comprehensive molecular portraits of human breast tumours. Nature 490: 61-70, 2012.

27. Pereira B, Chin SF, Rueda OM, Vollan HK, Provenzano E, Bardwell HA, Pugh M, Jones L, Russell R, Sammut SJ, et al: The somatic mutation profiles of 2,433 breast cancers refines their genomic and transcriptomic landscapes. Nat Commun 7: 11479, 2016.

28. Teramoto A, Shimazu K, Naoi Y, Shimomura A, Shimoda M, Kagara N, Maruyama N, Kim SJ, Yoshidome K, Tsujimoto M, et al: One-step nucleic acid amplification assay for intraoperative prediction of non-sentinel lymph node metastasis in breast cancer patients with sentinel lymph node metastasis. Breast 23: 579-585, 2014.

29. Maitra A, Wistuba II, Washington C, Virmani AK, Ashfaq R, Milchgrub S, Gazdar AF and Minna JD: High-resolution chromosome $3 p$ allelotyping of breast carcinomas and precursor lesions demonstrates frequent loss of heterozygosity and a discontinuous pattern of allele loss. Am J Pathol 159: 119-130, 2001.

30. Cornelisse CJ, van de Velde CJ, Caspers RJ, Moolenaar AJ and Hermans J: DNA ploidy and survival in breast cancer patients. Cytometry 8: 225-234, 1987.

31. Kimmig R, Wimberger P, Kapsner T and Hillemanns P: Flow cytometric DNA analysis using cytokeratin labeling for identification of tumor cells in carcinomas of the breast and the female genital tract. Anal Cell Pathol 22: 165-178, 2001.

32. Dayal JH, Sales MJ, Corver WE, Purdie CA, Jordan LB, Quinlan PR, Baker L, ter Haar NT, Pratt NR and Thompson AM: Multiparameter DNA content analysis identifies distinct groups in primary breast cancer. Br J Cancer 108: 873-880, 2013.

33. Galimberti V, Cole BF, Viale G, Veronesi P, Vicini E, Intra M, Mazzarol G, Massarut S, Zgajnar J, Taffurelli M, et al: Axillary dissection versus no axillary dissection in patients with breast cancer and sentinel-node micrometastases (IBCSG 23-01): 10-year follow-up of a randomised, controlled phase 3 trial, Lancet Oncol 19: 1385-1393, 2018.

34. Fujita N, Nakayama T, Yamamoto N, Kim SJ, Shimazu K, Shimomura A, Maruyama N, Morimoto K, Tamaki Y and Noguchi S Methylated DNA and total DNA in serum detected by one-step methylation-specific PCR is predictive of poor prognosis for breast cancer patients. Oncology 83: 273-282, 2012.

35. Chu PG and Weiss LM: Keratin expression in human tissues and neoplasms. Histopathology 40: 403-439, 2002.

36. Vilardell F, Novell A, Martin J, Santacana M, Velasco A, Díez-Castro MJ, Cuevas D, Panadés MJ, González S Llombart A, et al: Importance of assessing CK19 immunostaining in core biopsies in patients subjected to sentinel node study by OSNA. Virchows Arch 460: 569-575, 2012.

This work is licensed under a Creative Commons Attribution-NonCommercial-NoDerivatives 4.0 International (CC BY-NC-ND 4.0) License. 